\title{
Relationship between Blood Pressure Control Status and Lifestyle in Hypertensive Outpatients
}

\author{
Yuko Ohta, Takuya Tsuchihashi and Kanako Kiyohara
}

\begin{abstract}
Objective Lifestyle modification as well as combination antihypertensive therapy is necessary to achieve strict blood pressure (BP) control as advocated by the guidelines for the treatment of hypertension. The aim of this study was to investigate the status of adherence to lifestyle modifications and BP control status in hypertensive outpatients.

Methods and Patients Subjects are 661 hypertensive outpatients who had been followed at National Kyushu Medical Center. We assessed BP control status based on the average clinic BP on two occasions. In addition, we investigated the adherence to the individual items of lifestyle modification by a questionnaire.

Results Average BP was $129 \pm 10 / 71 \pm 11 \mathrm{mmHg}$ and overall rate of achieving goal BP was $60.1 \%$. Achieving rate of each target BP category was $83.3 \%$ in the elderly patients $(<140 / 90 \mathrm{mmHg}), 56.7 \%$ in the young/middle patients $(<130 / 85 \mathrm{mmHg})$ and $45.5 \%$ in the patients with diabetes mellitus/chronic kidney disease/myocardial infarction $(<130 / 80 \mathrm{mmHg})$. Adherance to each item of lifestyle modification was as follows: Patients who answered to be conscious about salt restriction was $80.9 \%$, those with increased intake of fruits/vegetables was $79.0 \%$, reduced intake of cholesterol/saturated fatty acids was $67.9 \%$, presence of obesity was $37.7 \%$, daily exercise for $\geq 30$ min was $31.9 \%$, habitual alcohol intake was $38.0 \%$, habitual smoking was $9.8 \%$. Only $22.5 \%$ of the patients had no lifestyle items to be modified. On the other hand, $19.6 \%$ of patients had more than 3 items to be modified. Subjects with more than 3 lifestyle items to be modified are more frequently found in young, male, and obese groups.

Conclusion We conclude that about $60 \%$ of the patients achieved goal BP by the intensive combination therapy. The lifestyle modification seems to be important especially for the young, male and obese patients.
\end{abstract}

Key words: BP control, combination antihypertensive therapy, diuretics, lifestyle modification, adherence

(Intern Med 50: 2107-2112, 2011)

(DOI: 10.2169/internalmedicine.50.5321)

\section{Introduction}

Blood pressure (BP) control was reported to be inadequate on the basis of either the routine clinic BP or home BP measurement (1-3). The Japanese Society of Hypertension guideline (JSH 2009) emphasizes that the use of two or three drugs including a low dose of diuretic is necessary to achieve the target BP (4). Genetic susceptibility as well as environmental factors related to lifestyle are involved in the development of hypertension. The effectiveness of lifestyle modification on the prevention and management of hypertension has been clearly shown in clinical trials $(5,6)$. Thus, lifestyle modification should be indicated for all hypertensive subjects $(4,7,8)$. In daily clinical practice, it seems to be important to recognize the lifestyle problems of the patients to be modified.

The aim of this study was to investigate the status of adherence to lifestyle modifications based on self-reporting questionnaire and BP control status in hypertensive outpatients.

\section{Materials and Methods}

Subjects were 661 hypertensive outpatients (362 females and 299 males, mean age $66 \pm 12$ years), who had been fol- 

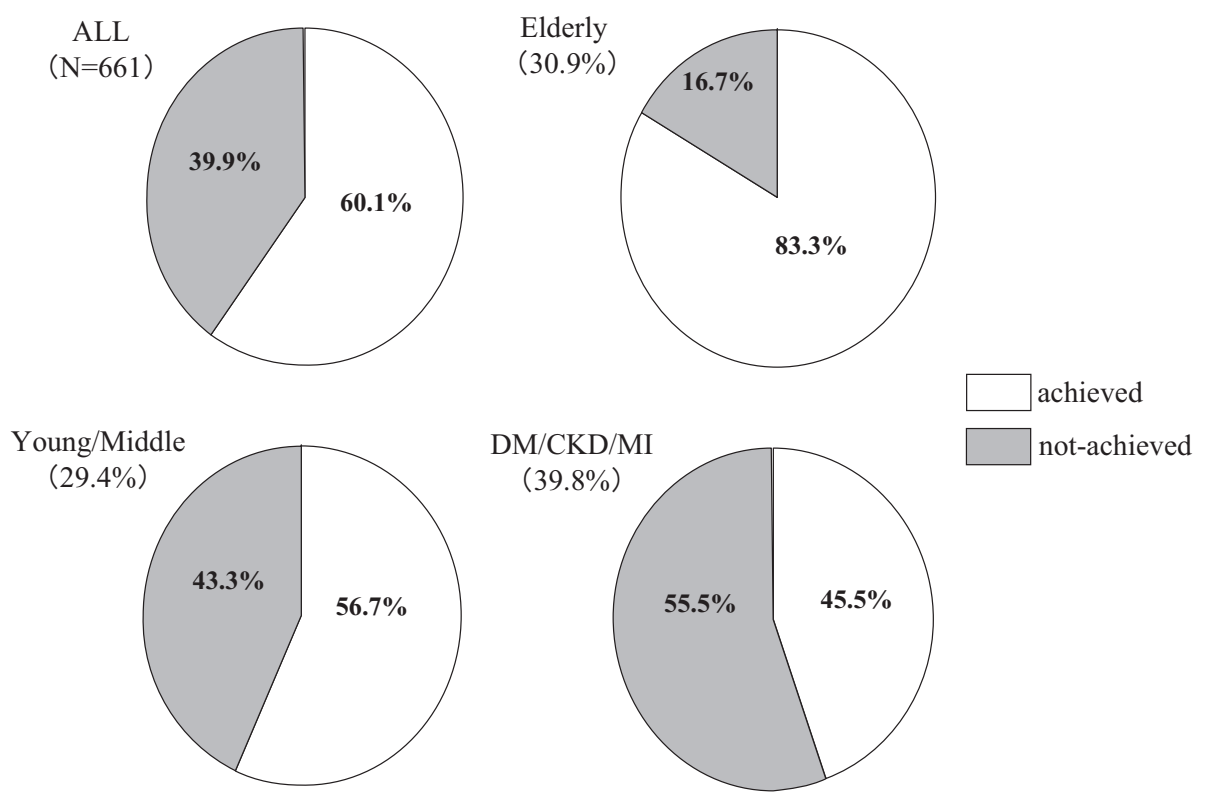

Figure 1. Blood pressure control status in all treated hypertensive patients, elderly patients, young/middle patients and the patients with DM/CKD/MI. DM: diabetes mellitus, CKD: chronic kidney disease, MI: myocardial infarction

lowed at National Kyushu Medical Center in Fukuoka, Japan. Patients with secondary hypertension such as renovascular hypertension and primary aldosteronism were excluded from the study and all patients took antihypertensive medicines for more than 3 months. We assessed BP control status based on the average clinic BP (CBP) on two occasions between May and June in 2010. CBP was measured with a sphygmomanometer by a doctor while the patient was seated. According to JSH 2009, goal BP was defined as SBP of $<140 \mathrm{mmHg}$ and DBP of $<90 \mathrm{mmHg}$ in elderly patients ( $\geq 65$ years), SBP of $<130 \mathrm{mmHg}$ and DBP of $<85$ $\mathrm{mmHg}$ in young or middle age patients, SBP of $<130$ $\mathrm{mmHg}$ and DBP of $<80 \mathrm{mmHg}$ in the patients with diabetes mellitus (DM) or chronic kidney disease (CKD) or myocardial infarction (MI). Body mass index (BMI) was calculated as weight $/$ height $^{2}\left(\mathrm{~kg} / \mathrm{m}^{2}\right)$. Obesity was defined as having BMI $\geq 25 \mathrm{~kg} / \mathrm{m}^{2}$. DM was defined as fasting serum glucose $\geq 126 \mathrm{mg} / \mathrm{dL}$, serum glucose $\geq 200 \mathrm{mg} / \mathrm{dL}$ at any time, HbA1c $\geq 6.5 \%$, or the current use of hypoglycemic agents. CKD was considered to be present if the patient had either decreased estimated glomerular filtration ratio (eGFR) $(<60$ $\mathrm{mL} / \mathrm{min}$ ) or persistent proteinuria. eGFR was calculated using the following formula: for men, 194× serum creatinine levels ${ }^{-1.094} \times$ age $^{-0.287}$; for women, $194 \times$ serum creatinine levels ${ }^{-1.094} \times$ age $^{-0.287} \times 0.739$ (9). MI was diagnosed based on medical history as well as the findings of electrocardiogram, echocardiography or coronary angiography. In addition, we investigated the patient's adherence to the individual item of lifestyle modification by a questionnaire.

This study was conducted following the guidelines of the National Kyushu Medical Center.

\section{Statistical analysis}

Values are presented as the mean \pm standard deviation (SD). The differences in the variables were compared by one-way ANOVA followed by the Scheffe's multiple comparison test when necessary. A chi-square test was also utilized when appropriate. $\mathrm{P}$ values less than 0.05 were considered significant.

\section{Results}

In all subjects, average BP in 2010 was $129 \pm 10 / 71 \pm 11$ $\mathrm{mmHg}$ and the proportion of achieving goal BP was $60.1 \%$ (Fig. 1). In the elderly patients $(<140 / 90 \mathrm{mmHg}), 83.3 \%$ achieved their goal BP, followed by $56.7 \%$ in the young/ middle patients $(<130 / 85 \mathrm{mmHg})$, and $45.5 \%$ in the patients with $\mathrm{DM} / \mathrm{CKD} / \mathrm{MI}(<130 / 80 \mathrm{mmHg})$. The average number of antihypertensive drugs was $2.3 \pm 1.0$ in all subjects, $2.1 \pm$ 1.0 in elderly, $2.0 \pm 0.9$ in young/middle and $2.6 \pm 1.1$ in $\mathrm{DM} / \mathrm{CKD} / \mathrm{MI}$. Ca channel blockers and angiotensin II receptor blockers (ARB) were mainly prescribed in all BP category groups. Diuretics were prescribed in $20.1 \%$ and there were no significant differences in the use of diuretics between patients with and without DM (22.5\% vs.19.8\%, ns). For the diuretics, $57 \%$ were provided as fixed-dose combination tablets with $\mathrm{ARB}$.

The items of lifestyle modification advocated by JSH 2009 are shown in Table 1. Adherence to each item of lifestyle modification is shown in Fig. 2. The percentage of patients who responded that they were conscious about salt restriction was $80.9 \%$, those with increased intake of fruits/ vegetables was $79.0 \%$, reduced intake of cholesterol/saturated fatty acids was $67.9 \%$, presence of obesity was $37.7 \%$, 
Table 1. Items of Lifestyle Modifications for Hypertensive Subjects (4)

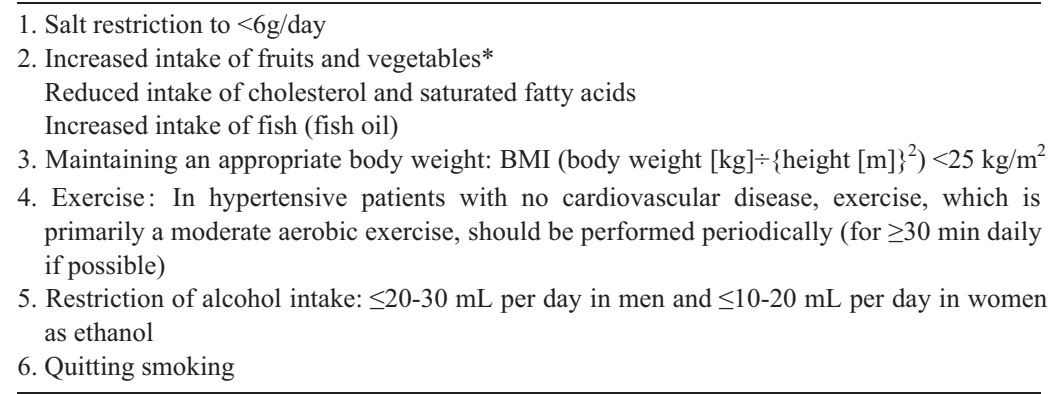

Comprehensive lifestyle modifications are more effective.

* An increased intake of fruit and vegetables is not recommended for patients with severe renal dysfunction because of the risk of hyperkalemia. An excessive intake of fruit with a high fructose content is not recommended in patients who need to undergo a restricted energy intake, such as obese and diabetic patients.

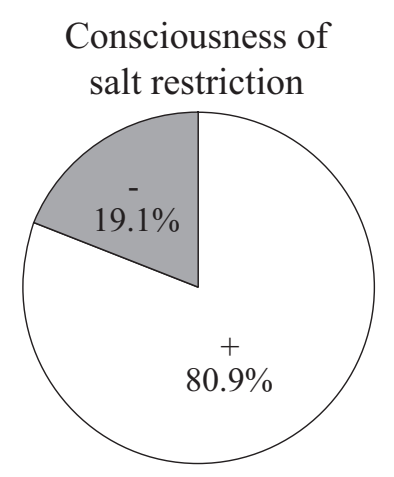

$$
\begin{aligned}
& \text { Increased intake of } \\
& \text { fruits/vegetables }
\end{aligned}
$$

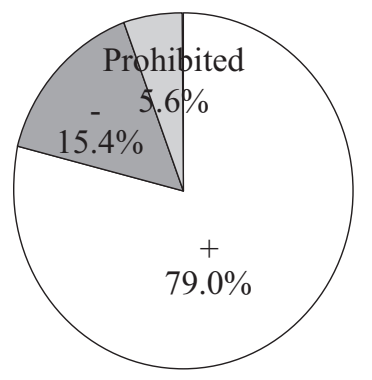

Reduced intake of

cholesterol/saturated fatty acids

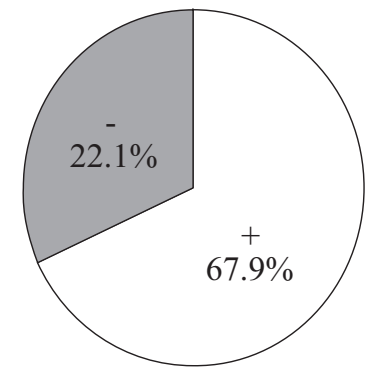

Daily exercise

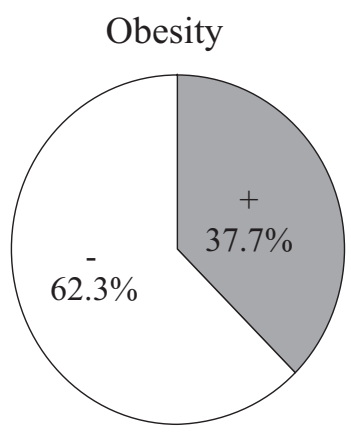

for $\geq 30 \mathrm{~min}$

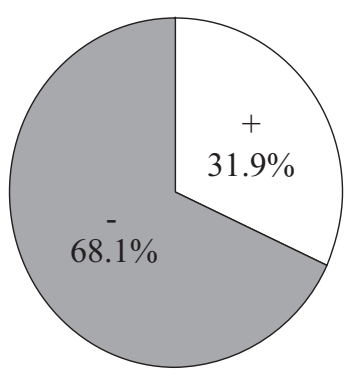

Habitual alcohol intake

Habitual smoking

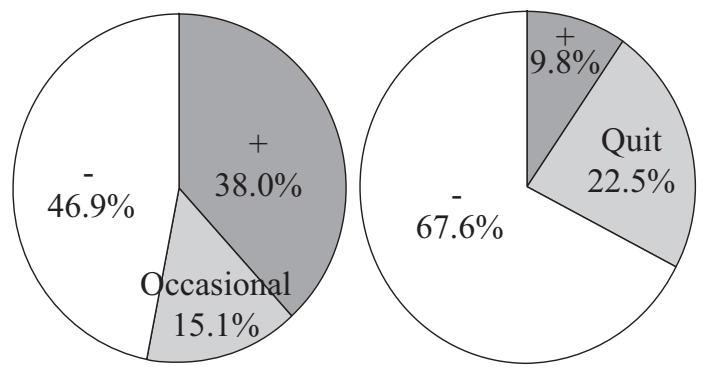

Figure 2. Adherence to each item of lifestyle modification advocated by JSH 2009. Results on salt restriction, increased intake of fruits/vegetables, reduced intake of cholesterol/saturated fatty acids, presence of obesity, daily exercise for $\geq 30 \mathrm{~min}$, habitual alcohol intake and habitual smoking are shown.

daily exercise for $\geq 30$ min was $31.9 \%$. The patients with habitual alcohol intake and habitual smoking were $38.0 \%$ and $9.8 \%$, respectively. Figure 3 shows the relationship between consciousness of salt restriction and other lifestyle items. The patients who were not-conscious of salt restriction also showed lesser adherence to the intake of fruit/vegetables, reduction of cholesterol/saturated fatty acids and habitual exercise. Habitual alcohol intake was also more prevalent among them.

Only $22.5 \%$ of the patients had no lifestyle items to be modified (Fig. 4). On the other hand, 32.0\% of patients had one, $25.9 \%$ of patients had two, and $19.6 \%$ of patients had more than three items to be modified. Subjects with more than 3 lifestyle items to be modified were found to be more frequently in young, male, and obese groups (Fig. 5). The patients with uncontrolled BP tended to be more prevalent in the patients having more than 3 problems $(p=0.09)$.

\section{Discussion}

The present study indicates that the proportion of achieving goal BP is $60.1 \%$ in all subjects. In our hypertensive patients, the proportion of achieving goal BP significantly increased from 2008 to 2010 . In addition, the prescription of diuretics was increased during this period and $57 \%$ of diuretics were provided as fixed-dose combination tablets with 


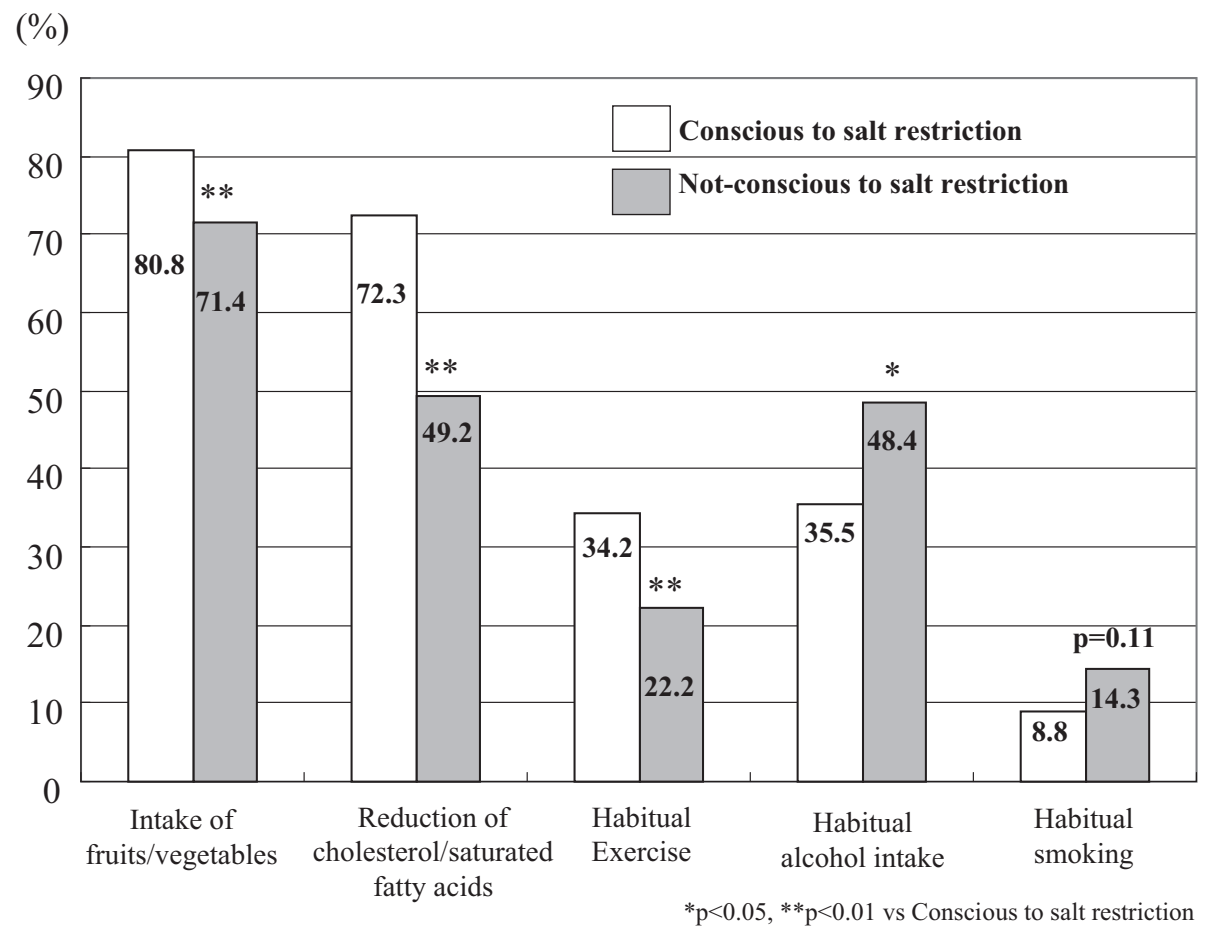

Figure 3. Relationship between consciousness of salt restriction and other lifestyle items, such as increased intake of fruits/vegetables, reduced intake of cholesterol/saturated fatty acids, daily exercise for $\geq \mathbf{3 0}$ min, habitual alcohol intake and habitual smoking.
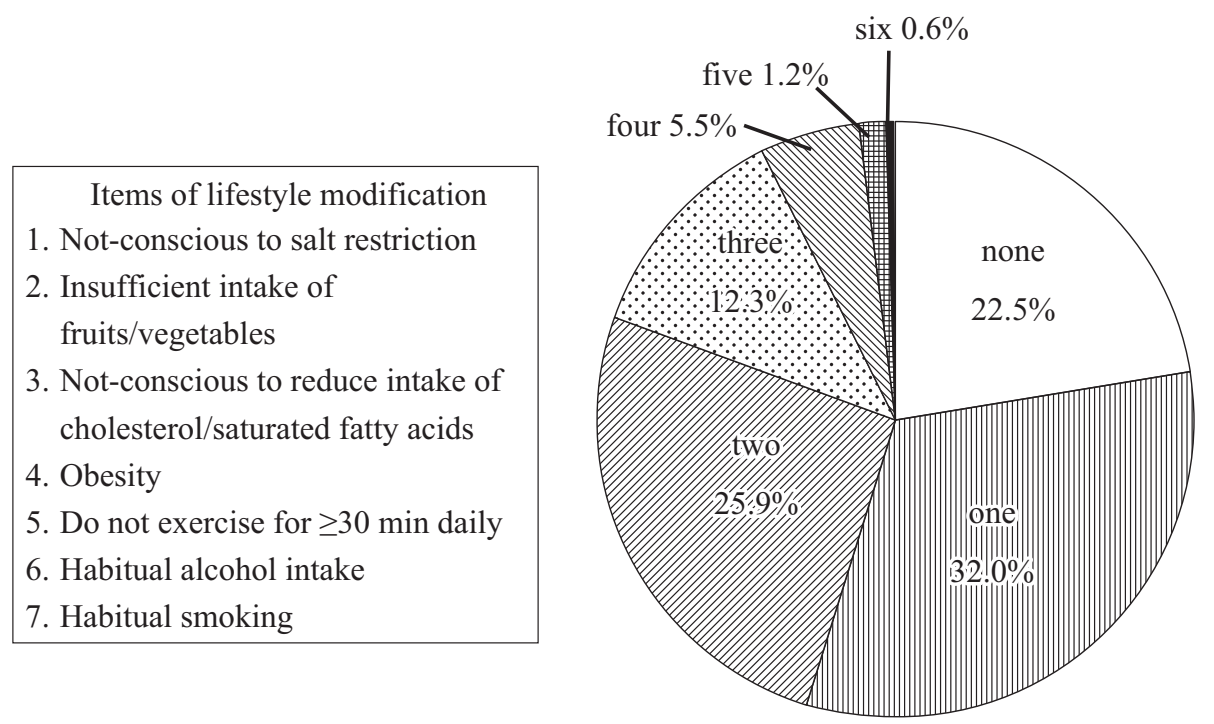

Figure 4. Number of lifestyle items to be modified.

ARB. (unpublished data). The prevalence of patients with achieving goal BP seemed to increase by the intensive antihypertensive combination therapy including diuretics advocated by the guidelines in the present study. Many guidelines for the management of hypertension, such as JNC 7, ESH/ESC 2007 and JSH 2009 guidelines recommend intensive antihypertensive treatment, in particular, the use of diuretics when three or more antihypertensive drugs are required $(4,7,8)$. However, the frequency of hypertensive patients treated with diuretics is reported to be low in Japan, being less than $10 \%$. It is well known that diuretics have several adverse effects, such as hypokalemia, hyperuricemia, dyslipidemia, and impairment of glucose metabolism in addition to volume depletion (10). However, diuretics seem to be effective for the treatment of Japanese hypertensive patients because the salt intake of Japanese remains to be high. Therefore, the use of low-dose thiazide diuretics is recommended to obtain sufficient antihypertensive effects with minimal metabolic adverse effects $(11,12)$. Fixed-dose combination tablets of ARB with low dose diuretics became available in Japan, which may facilitate the use of diuretics in the process of achieving goal BP. Indeed, the fixed-dose 


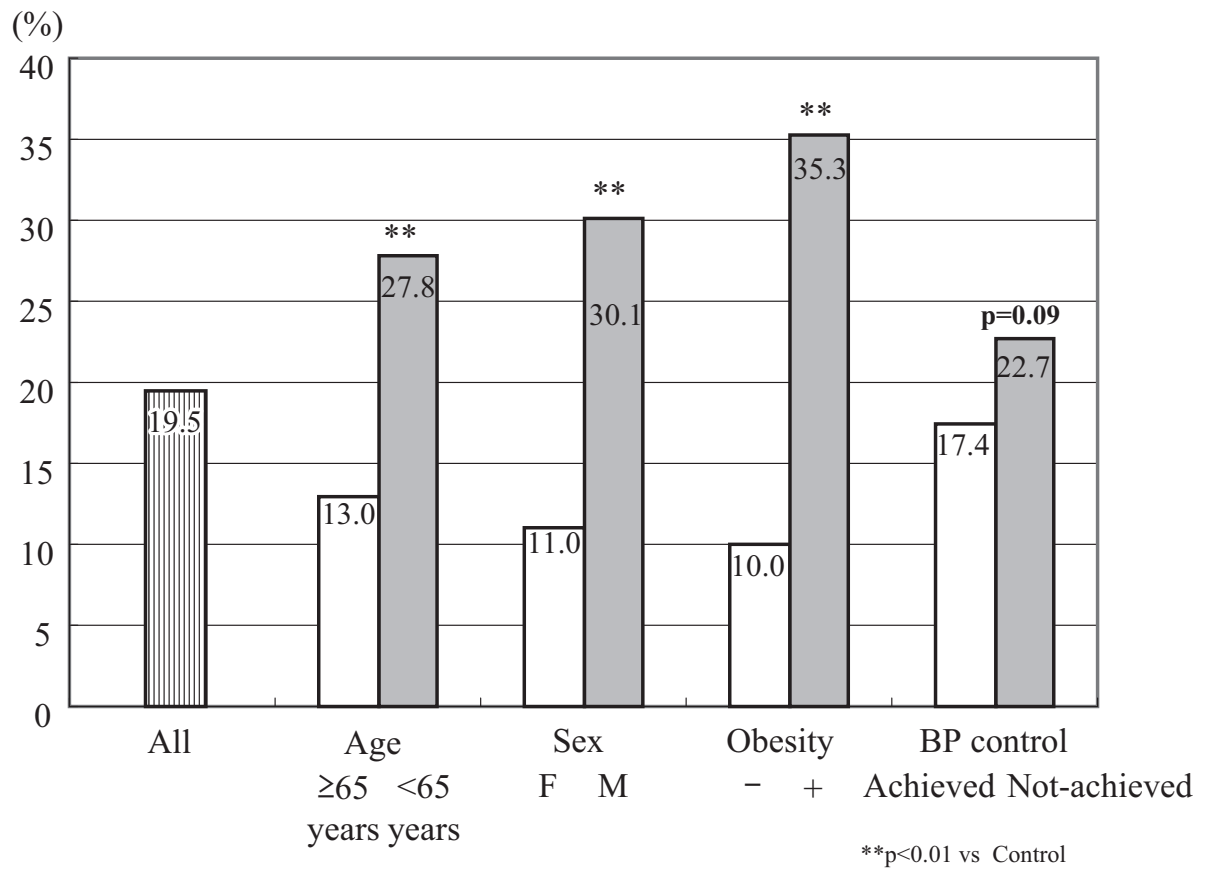

Figure 5. Frequency of patients with more than three lifestyle items to be modified. F: female, M: male

combination drug was used in $57 \%$ of the patients taking diuretics in the present study. The use of fixed-dose combination drugs is advantageous for improving adherence by reducing the number of tablets to be taken (13). However, poor BP control is exclusively found in the young/middle patients or those with DM/CKD/MI for whom low goal BP was advocated. Thus, more aggressive antihypertensive therapy seems to be needed in the young/middle patients or those with DM/CKD/MI.

Another finding of the present study was that subjects with more than 3 lifestyle items to be modified are more frequently found in young, male, and obese groups. The patients with uncontrolled BP also tended to be more prevalent in those with 3 or more lifestyle problems. It is obvious that salt restriction is the most important issue. Indeed, $80.9 \%$ of the patients answered to be conscious about salt restriction in this study. However, we have previously reported that 24$\mathrm{h}$ urinary salt excretion is similar between the salt-conscious and salt-unconscious patients (14), suggesting that monitoring the salt excretion is quite important to confirm salt intake of the individual patients. Since long-term compliance of salt restriction is poor in Japanese hypertensive patients $(3,15)$, the encouragement of other lifestyle modification such as body weight reduction as well as the intensive antihypertensive treatment is required to achieve goal BP (3). The effectiveness of sodium reduction program in combination with weight loss in the treatment of hypertension was also confirmed by the TONE study (6). Visceral obesity, which is closely related to metabolic syndrome, induces not only hypertension but also abnormalities of glucose and lipid metabolism (16). We have previously reported that patients with metabolic syndrome show higher urinary salt excretion and need more antihypertensive drugs to manage their BP (17). Habitual drinking, male gender and older age are also reported to associate with persistently uncontrolled home BP $(2,18)$. Since marked BP reduction can be achieved by a combination of salt restriction, weight loss, exercise, restriction of alcohol intake, and a DASH diet (19), comprehensive lifestyle modifications seem to be important for achieving BP control in hypertensive patients, in particular, young male patients with obesity.

The limitation of the present study is that the subjects are hypertensive outpatients who have been followed at a specialized hypertension clinic and the investigation on lifestyle modifications is based on self-reporting. Therefore, the present findings may not indicate the precise current status of lifestyle modification and BP control in the hypertensive patients of Japanese general population. In addition, we have not assessed the doses of antihypertensive medicines. However, JSH 2009 recommends the use of two or three drugs including low dose of diuretics. The low number of antihypertensive drugs seems to contribute to poor BP control.

In conclusion, about $60 \%$ of the patients achieved goal BP by the intensive combination therapy. The young, male and obese patients had more lifestyle items to be modified, suggesting the importance of leading these patients to modify individual lifestyle items.

The authors state that they have no Conflict of Interest (COI).

\section{References}

1. Hozawa A, Ohkubo T, Kikuya M, et al. Blood pressure control assessed by home, ambulatory and conventional blood pressure 
measurements in the Japanese general population: the Ohasama study. Hypertens Res 25: 57-63, 2002.

2. Ohta Y, Tsuchihashi T, Kiyohara K. Consequence of masked hypertension in treated hypertensive outpatients: one-year follow-up study. Clin Exp Hypertens 33: 270-274, 2011.

3. Ohta Y, Tsuchihashi T, Onaka U, Miyata E. Long-term compliance of salt restriction and blood pressure control status in hypertensive outpatients. Clin Exp Hypertens 32: 234-238, 2010.

4. Ogihara T, Kikuchi K, Matsuoka H, et al; on behalf of the Japanese Society of Hypertension Committee for guidelines for the management of hypertension. The Japanese Society of Hypertension guidelines for the management of hypertension (JSH 2009). Hypertens Res 32: 4-107, 2009.

5. Appel LJ, Moore TJ, Obarzanek E, et al; DASH Collaborative Research Group. A clinical trial of the effects of dietary patterns on blood pressure. N Engl J Med 336: 1117-1124, 1997.

6. Whelton PK, Apple LJ, Espeland MA, et al; TONE Collaborative Research Group. Sodium reduction and weight loss in the treatment of hypertension in older persons: a randomized controlled trial of nonpharmacologic interventions in the elderly (TONE). JAMA 279: 839-846, 1998.

7. Chobanian AV, Bakris GL, Black HR, et al. Joint National Committee on Prevention, Detection, Evaluation, and Treatment of High Blood Pressure. National Heart, Lung, and Blood Institute; National High Blood Pressure Education Program Coordinating Committee: Seventh report of the joint national committee on prevention, detection, evaluation, and treatment on high blood pressure. Hypertension 42: 1206-1252, 2003.

8. 2007 guidelines for the management of arterial hypertension. The task force for the management of arterial hypertension of the European Society of Hypertension (ESH) and of the European Society of Cardiology (ESC). J Hypertens 25: 1105-1187, 2007.

9. Matsuo S, Imai E, Horio M, et al. Collaborators developing the Japanese equation for estimated GFR: Revised equations for estimated GFR from serum creatinine in Japan. Am J Kidney Dis 53: 982-992, 2009.

10. Plavinik FL, Rodrigues CI, Zanella MT, Ribeiro AB. Hypoka- lemia, glucose intolerance, and hyperinsulinemia during diuretic therapy. Hypertension 19: S1126-S1129, 1992.

11. Saruta T, Ogihara T, Matsuoka $\mathrm{H}$, et al. Antihypertensive efficacy and safety of fixed-dose combination therapy with losartan plus hydrochlorothiazide in Japanese patients with essential hypertension. Hypertens Res 30: 729-739, 2007.

12. Law MR, Wald NJ, Morris JK, Jordan RE. Value of low dose combination treatment with blood pressure lowering drugs: analysis of 354 randomised trials. BMJ 326: 1427, 2003.

13. Bangalore S, Kamalakkannan G, Parkar S, Messerli FH. Fixeddose combinations improve medication compliance: a metaanalysis. Am J Med 120: 713-719, 2007.

14. Ohta Y, Tsuchihashi T, Ueno M, Onaka U, Tominaga M, Eto K. Relationship between the awareness of salt restriction and the actural salt intake in hypertensive patients. Hypertens Res 27: 243246, 2004.

15. Ohta Y, Tsuchihashi T, Onaka U, Eto K, Ueno M. Long-term compliance of salt restriction in Japanese hypertensive patients. Hypertens Res 28: 953-957, 2005.

16. Committee to Evaluate Diagnostic Standards for Metabolic Syndrome. Nippon Naika Gakkai Zasshi 94: 794-809, 2005 (in Japanese).

17. Ohta Y, Tsuchihashi T, Arakawa K, Onaka U, Ueno M. Prevalence and lifestyle characteristics of hypertensive patients with metabolic syndrome followed at an outpatient clinic in Fukuoka, Japan. Hypertens Res 30: 1077-1082, 2007.

18. Ishikawa J, Kario $\mathrm{K}$, Eguchi $\mathrm{K}$, et al; on behalf of the J-MORE Group. Regular alcohol drinking is a determinant of masked morning hypertension detected by home blood pressure monitoring in medicated hypertensive patients with well-controlled clinic blood pressure: The Jichi Morning Hypertension Research (JMORE) study. Hypertens Res 29: 679-686, 2006.

19. Apple LJ, Champagne CM, Harsha DW, et al; Writing Group of the PREMIER Collaborative Research Group. Effects of comprehensive lifestyle modification on blood pressure control: main results of the PREMIER clinical trial. JAMA 289: 2083-2093, 2003.

(C) 2011 The Japanese Society of Internal Medicine http://www.naika.or.jp/imindex.html 